\title{
Systematic Innovation Practice for Enhanced Mobile Advertisement Protocol Design
}

\author{
Kim Song-Kyoo, Senior Member, IEDRC
}

\begin{abstract}
The paper deals Systematic Innovation method adaptations for revealing the mobile technology applications. MobAD enabler can provide the usage of a variety of advertisement delivery methods and also specify a standard set of advertisement metrics data that are recorded and then collected to enable the measurement of the response to advertise contents and campaigns. MobAD regional code is the new MobAD technology that is designed to give flexible operations by using the systematic method for the revenue generating of mobile operators.
\end{abstract}

Index Terms-Innovation, MobAD, OMA, regional code, TRIZ, TIPS, systematic innovation.

\section{INTRODUCTION}

Mobile Advertisement (MobAD) is considered as the target to apply the systematic innovation for enhancement because MobAD is one of popular features in the mobile industries and actively involved in mobile standard activities. The MobAD takes advantage of information available about mobile device users and how they use their devices to provide the users with advertisements that are closely targeted to their interests, leading to high advertisement response rates. It is designed for supporting the successful deployment of mobile advertising services by providing an inter-operable framework for adding the personalization, delivery and metrics data collection [1]. The MobAD enabler also specifies a standard set of advertisement metrics data that are recorded (as users view ads and interact with them) and then collected to enable the measurement of the response to ads and ad campaigns. The MobAD enabler can make usage of a variety of advertisement delivery methods, (e.g. pull, push and broadcast delivery).

The systematic innovation method could be the recursive process and it is also a set of continuous evolving tools that will improve ability to solve the problems. TRIZ (TIPS; Theory of Inventive Problem Solving) is the most powerful tool set for systematic innovation [2], [3]. TRIZ (Teoriya Resheniya Izobretatelskikh Zadatch) that is also called TIPS is a methodology and model-based technology for generating innovative ideas and solutions for problem solving [4], [5]. It has been developed by Genrich Altshuller who have believed that learning how to invent is new tings [4]-[6]. After his first approach, TRIZ has been evolved as the science of innovation and many companies are adopting TRIZ to solve complex technical problems. TRIZ methodology is applied

Manuscript received August 30, 2014; revised October 23, 2014.

Song-Kyoo Kim is with Asian Institute of Management, Makati City 1260, Philippines (e-mail: SKim@aim.edu). for mobile technology enhancement of MobAD standard in OMA [6]. TRIZ adaptation in mobile industries has been proposed by author [7]. This research is another tactical TRIZ approach to demonstrate the guidelines for applying into the information technologies (see Fig. 1).

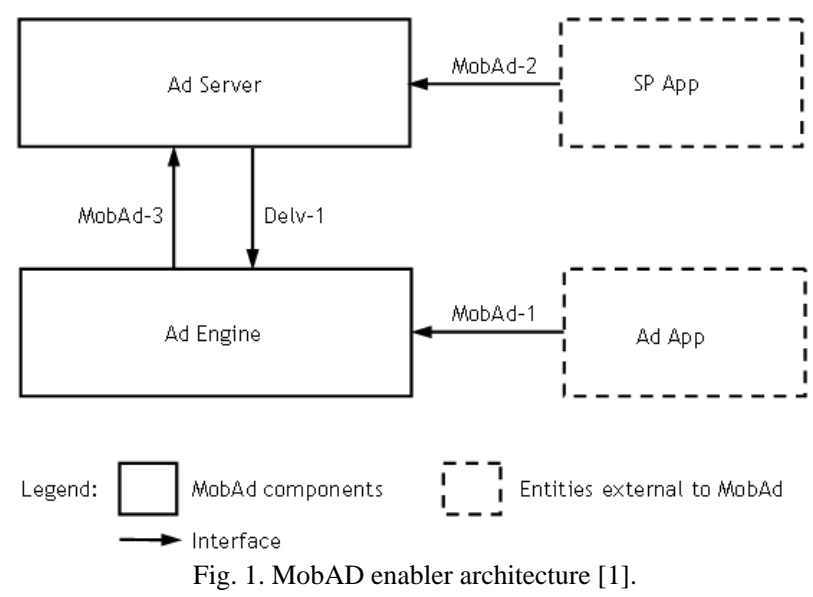

\section{GENERAL FRAMEWORK FOR SYSTEMATIC INNOVATION FRAMEWORK}

The framework of the systematic innovation by using the enhanced Su-Field notations [8] has been introduced on 2002 and it has been widely applied. This section is basically adapting the previous research by the author [9]. Ideal final result (IFR) is a description of the best possible solution for the problem situation (or contradiction), regardless of the resources or constraints of the original problem. A well-defined IFR helps a problem solver to reach breakthrough solutions by thinking about the solution in terms of functions, not the intervening problems or needed resources [9]. It focuses on functions needed, not the current process or equipment. It is therefore the antithesis to the more commonly used continuous improvement method which often leads to progressively diminishing returns (the classic S-curve). IFR represents a significant shift in the thinking approach to solving problems [9].

$$
\text { Ideality }=\frac{\sum_{i}^{\infty} U_{i}}{\sum_{j}^{\infty} H_{j}}
$$

The concept design of the new implementations based on Su-Field model could be determined couple for solutions within 76 Inventive Standard solutions. The Su-Field model 
for Inventive standard solution can exhibits the summarized main characteristics of a Su-Field model which adapted from Kim [8]:

$$
(x / s / f):(/ a)
$$

where the symbols $x, s, f$ and $a$ stand for basic elements of the model as follows:

$$
x=\text { solution (or problem) types }(x=1,2 \text { or } 4) \text {; }
$$

$s=$ substance attributes;

$f=$ field attributes;

$a=$ strength of actions ( $a=0 ;$ Normal or $a=1 ;$ Stronger $)$;

The attributes of the substance $S$ are as follow:

$S^{*}=$ general terms of the substance that can solve the problems;

$S^{+}=+1$ substance from basic structure to solve the problems;

$S^{\prime}=$ modify the substance (tool) to solve the problems without changing the number of components from basic structure;

$\mathrm{S}^{-}=-1$ substance from basic structure (i.e., tool is missed)

$S^{\infty}=$ substance (tool) is divided infinitely (Technical System Evolution);

$S$ " or $S^{2}=$ adding the clone of the substance $(+1)$.

The attributes of the field $f$ are similar with substance attributes:

$F^{*}=$ general terms of the field that can solve the problems;

$F^{+}=+1$ field from basic structure to solve the problems;

$F^{\prime}=$ modify the field to solve the problems without changing the number of components from basic structure;

$F^{-}=-1$ field from basic structure;

$F^{\infty}=$ field is divided infinitely (Technical System Evolution);

$F^{\prime \prime}=$ adding the clone of the field $(+1)$;

$\leftarrow$

$\overleftarrow{F}=$ reverse direction of the field.

The attributes for fields and substances indicate how to modify the substances and the fields. According to the enhanced Su-Field model [8], the concept problems for the effective MobAD implementations are either Type-1 or Type-2 problems:

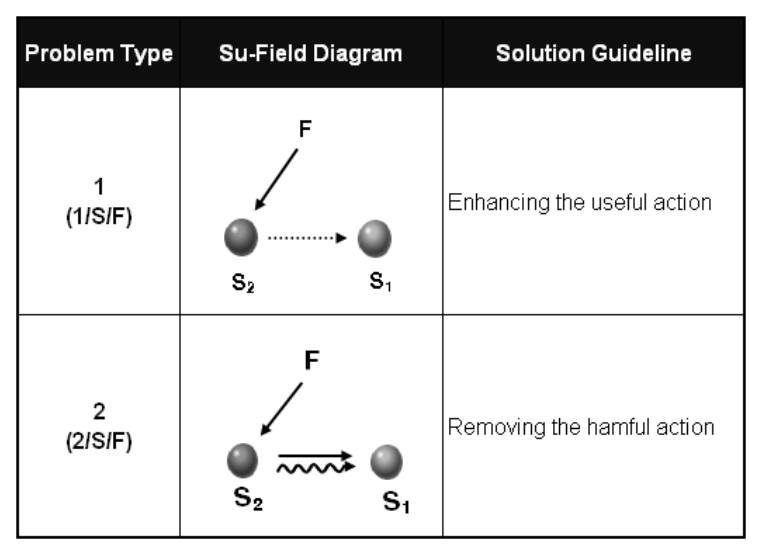

Fig. 2. Problem types in the enhanced Su-Field notations.

The notation method is clarifying the Inventive Standards simpler ways and users can be guided to the candidate solutions from the problems based on Su-Field model with the minimal knowledge of 76 Inventive Standard solutions.
The new notation for Su-Field model (Su-Field notation) is provided [8]. The Su-Field model for Inventive standard solution can exhibits the summarized main characteristics of a Su-Field model (see Fig. 2)..

$$
x / S^{\{y\}} / F^{\{z\}} \rightarrow \begin{cases}1 / S^{\{*\}} / F^{\left.f^{*}\right\}}, & x=1, \\ 2 / S^{\left.{ }^{*}\right\}} / F^{\left\{^{*}\right\}}, & x=2, \\ 4 / S^{\{*\}} / F^{\left\{^{*}\right\}}, & x=4\end{cases}
$$

where

$$
S^{\{*\}}=\left\{S^{+}, S^{\prime}, S^{\infty}\right\}
$$

The solutions are equivalent with 1-1-3, 1-2-1, 1-2-2 and 2-2-2 within 76 Inventive Standard Solutions [9].

\section{INNOVATIVE MOBAD ENHANCEMENT}

In the case of MobAD, the constraints based on physical domain is not the scope of current OMA standard. But it needs to be discussed because the advertisement contents distribution based on domain can control the traffic cost. The metadata that contains regional indicator is called regional code and the technical contradictions (TC) are:

TC-1: If MobAD contents are pushed to users on roaming area then good for exposing Ad contents, but bad for increasing the roaming charge.

TC-2: If MobAD contents are NOT pushed to users, then good for removing the roaming charge.

TC-1 is taken to design the regional code in MobAD metadata (see Fig. 3- Fig. 4). Idle Final Result (IRF) can be defined as good for selected exposing MobAD contents based on regional restrictions. Based on the above conditions, Invention principle 10 (Preliminary action) and Invention principle 19 (Periodic action) are applied to solve the technical contradiction [4].

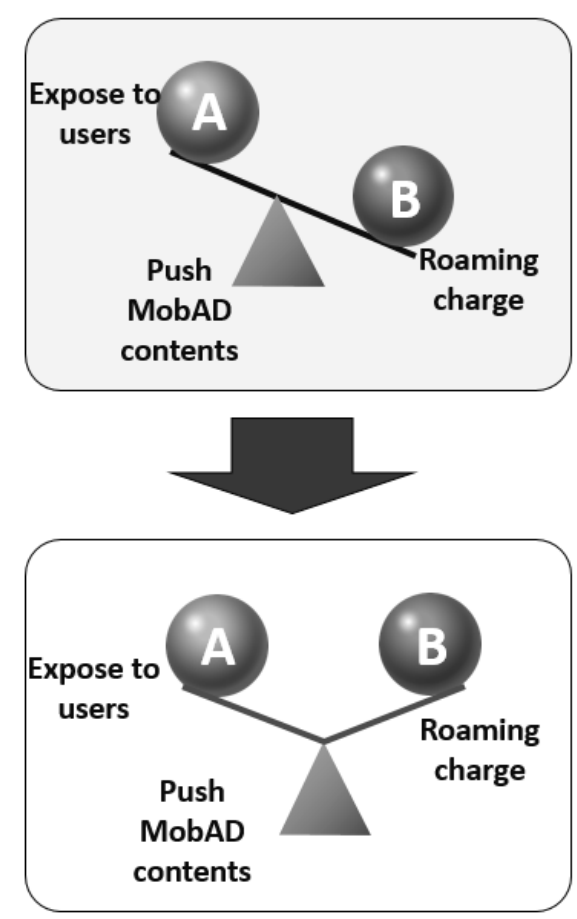

Fig. 3. Idle final result of TC-1. 


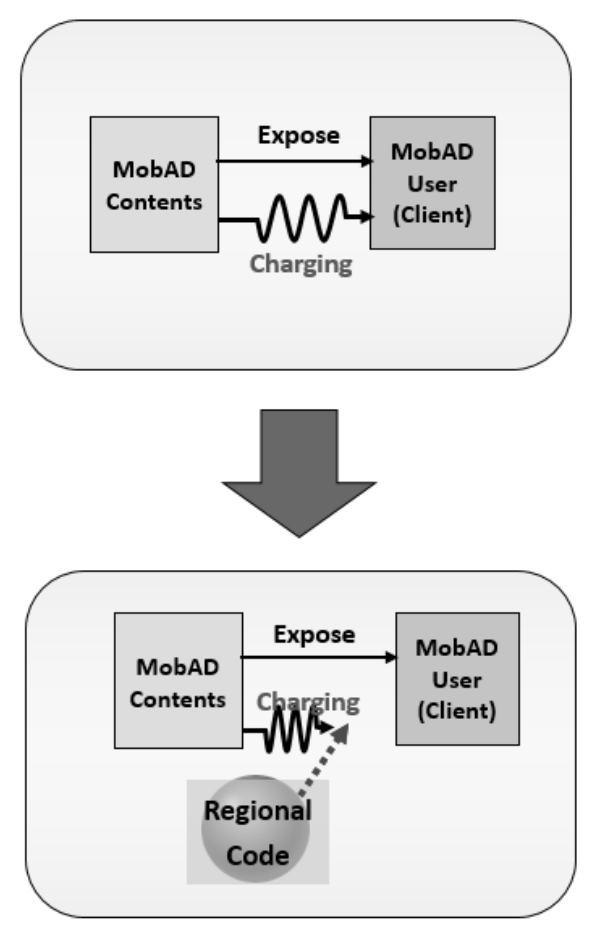

Fig. 4. The solution of the enhanced MobAD $\left(2 / S^{+} / F\right)$.

There is another TRIZ approach to solve the roaming expenses of MobAD contents. The enhanced Substance-Field (Su-Field) model is targeted for analyze the problems related existing technological systems [8]. Su-Field model analysis is applied for making innovative problems to the form of analysis model related to the current technical system. Based on the framework, this is the problem Type-2 $(2 / \mathrm{S} / \mathrm{F})$ and the guideline of the solution is removing the harmful effect $\left(2 / S / F \rightarrow 2 / S^{+} / F\right)$.

\section{Design of Regional Code for Mobile ADVERTISEMENT}

TABLE I: STRUCTURE OF MOBAD METADATA [10]

\begin{tabular}{|l|l|l|l|}
\hline \multicolumn{1}{|c|}{ Element } & Requirement & \multicolumn{1}{|c|}{ Type } & \multicolumn{1}{c|}{ Description } \\
\hline Regional Code & $\begin{array}{l}\text { Mandatory or } \\
\text { Blank }\end{array}$ & $\begin{array}{l}2 \text { digit } \\
\text { decimal } \\
\text { (call } \\
\text { number) or } \\
\text { 2 byte char. } \\
\text { (DNS) }\end{array}$ & $\begin{array}{l}\text {. Regional (country) code for Ad source } \\
\text { Regional information for destination (call number or DNS) } \\
\text { (ex., call number: } 49 \rightarrow \text { Germany, } 82 \rightarrow \text { Korea or } \\
\text { DNS: de } \rightarrow \text { germany, kr } \rightarrow \text { Korea, jp } \rightarrow \text { Japan) } \\
\text { Special code for wildcard } \\
\rightarrow \text { zZ (DNS), 99 (call number) or Blank: No regional restrction }\end{array}$ \\
\hline Ad ID & Mandatory & String & Unique identifier of the specific Advertisement requested \\
\hline Format & Mandatory & String & Identifies the associated Ad format \\
\hline $\begin{array}{l}\text { Type } \\
\text { Expiration Date, } \\
\text { Keyword, } \\
\begin{array}{l}\text { Targeted } \\
\text { Audience, }\end{array}\end{array}$ & Mandatory & String & Indicate the type of the Ad (e.g. banner) \\
\hline
\end{tabular}

The MobAD regional code define the constraint that domain of MobAD data. It reduce the unwanted extra costs such as data roaming.

The types of regional code are either 2 digit decimals for phone number or 2 bytes for DNS code (see Table I.) The main purpose of the regional codes is controlling the MobAD contents more effectively. The default case is no regional restrictions and operators or contents providers pay the traffic charge. Media gateway such as PPG (Push Proxy Gateway) and SIP gateway contains the module that can look up the metadata because it is plain code (i.e., not encrypted).

Mobile Advertising takes advantage of information available about mobile device users and how they use their devices to provide the users with advertisements that are closely targeted to their interests, leading to high advertisement response rates (see Fig. 5). The MobAD enabler will support the successful deployment of Mobile Advertising services by providing an interoperable framework for ad personalization, delivery and metrics data collection [10].

The MobAD enabler allows ad personalization by defining metadata about the target audience for an ad contents or campaign; this information can be matched against user profile and preference information so that the user only receives ad contents that are of interest to them. In other words, the localized advertisements from other regions were blocked because of the regional codes (see Fig. 6).

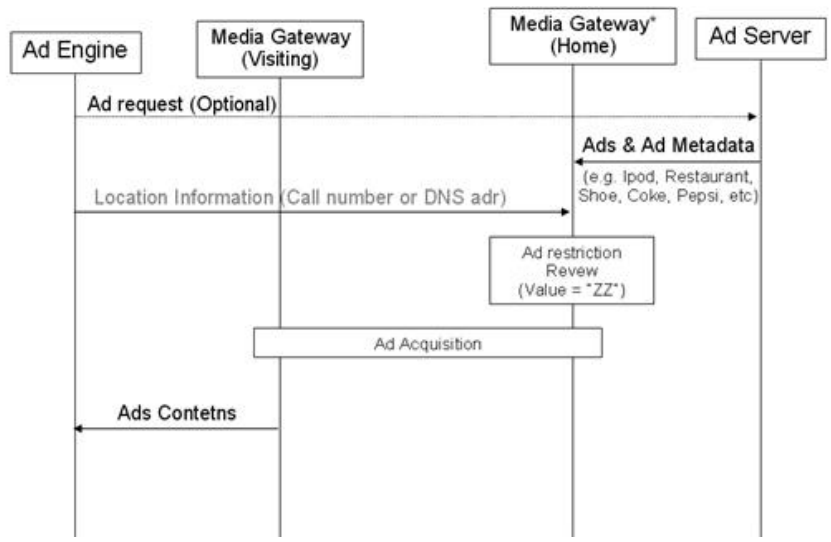

Fig. 5. Standard workflow of the mobile advertisement [10].

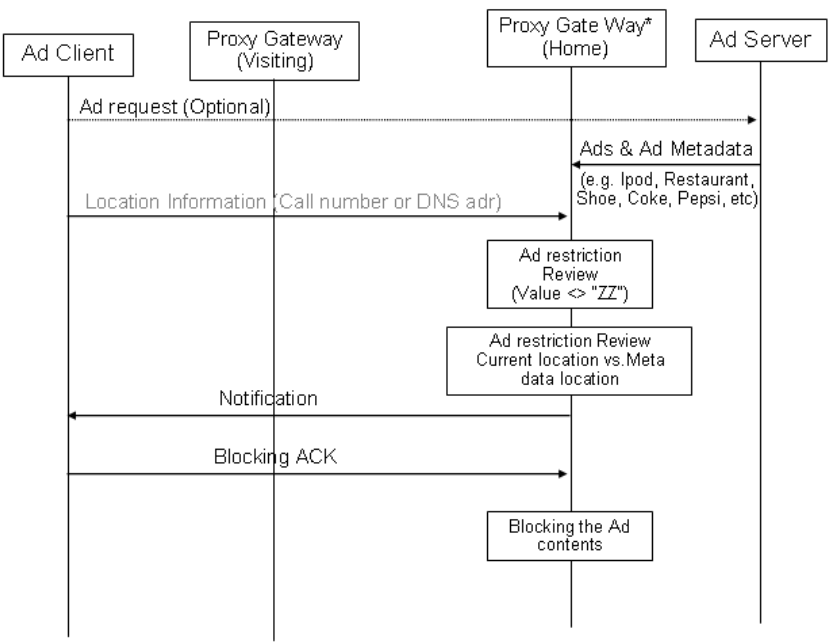

Fig. 6. Blocking the Ad contents based on regional code [10].

\section{CONCLUSION}

The major target of this project is developing the new MobAD constraints to control the MobAD contents based on regional codes. This new technology supports more flexible business models because advertisement is one of revenue generations for operators.

\section{REFERENCES}

[1] Open Mobile Alliance. (2009). OMA MobAD Requirement Document. [Online]. Available: http://www.openmobilealliance.org 
[2] E. Domb, "The seventy-six standard solutions: how they relate to the 40 principles of inventive problem solving," TRIZ Journal, vol. 2, 1999.

[3] F. Grace and et al., "A new TRIZ practitioner's experience for solving an industrial problem using ARIZ 85C," TRIZ Journal, vol. 1, 2001.

[4] G. Altshuller, And Suddenly the Inventor Appeared: TRIZ, the Theory of Inventive Problem Solving, Technical Innovation Center, Worecester, 1996.

[5] J. Terninko, A. Zusman et al., Systematic Innovation: An Introduction to Theory of Inventing Problem Solving, Boca Raton, FL: CRC Press, 1998.

[6] K. Rantanen and E. Domb, Simplified TRIZ, 1st ed., Boca Raton, FL: CRC Press, 2002.

[7] S. K. Kim, "Design of event driven Drm," in Proc. the International Conference on Industrial Engineering and Engineering Management, pp. 935-938, 2007.

[8] S. K. Kim, "Concept design based on substance-field model in theory of inventive problem solving," International Journal of Innovation Management and Technology, vol. 3, no. 4, 2012, pp. 306-309
[9] S. K. Kim, "General framework for user experience design based on systematic innovation," International Journal of Social Science and Humanity, vol. 3, no. 2, 2013, pp. 100-102.

[10] S. K. Kim, "System and method for providing region-based mobile advertising," US Patent , 2010.

Song-Kyoo Kim is an associate professor of Asian Institute of Management. $\mathrm{He}$ had been a technical manager and TRIZ specialist of mobile communication division at Samsung Electronics. He is involved in IT industries more than 10 years. Dr Kim has received his master degree of computer engineering in 1999 and Ph.D. of operations research in 2002 from Florida Institute of Technology. He is the author of more than 20 operations research papers focused on stochastic modeling, systematic innovations and patents. He had been the project leader of several 6 Sigma and TRIZ projects mainly focused on the mobile industry. 